\title{
A novel prediction model for diagnosing hepatocellular carcinoma in patients with chronic liver disease based on serological biomarkers
}

Jun Gao

Nanchang University Second Affiliated Hospital

Chunyan Xie

Nanchang University

Xin Yu

Nanchang University Second Affiliated Hospital

Yingbin Xiao

Nanchang University Second Affiliated Hospital

Xiaogang Peng

Nanchang University

Xiangbao Ying

Nanchang University Second Affiliated Hospital

Daojing Li

Jiangxi Cancer Hospital

Fan Zhou ( $\nabla$ nczhoufan@sina.cn )

Nanchang University https://orcid.org/0000-0003-2612-7950

Research article

Keywords: Hepatocellular carcinoma, 5-hydroxylmethylcytosine, AFP, diagnostic model

Posted Date: March 3rd, 2020

DOI: https://doi.org/10.21203/rs.3.rs-15901/v1

License: (1) (1) This work is licensed under a Creative Commons Attribution 4.0 International License.

Read Full License 


\section{Abstract}

Background: Hepatocellular carcinoma (HCC), the most common type of digestive cancers, is a common complication of chronic liver disease (CLD). Currently, HCC is often diagnosed by radiological examination. 5-Hydroxymethylcytosine $(5 \mathrm{hmC})$ in a vital mammalian DNA epigenetic modification that is found in circulating cell-free DNA (cfDNA) and carries tumor-specific epigenetic information. It may also serve as a potential cancer biomarker for HCC diagnosis. Our study aimed to establish a statistical diagnostic model for diagnosing the risk of HCC in individual patients with CLD according objective tests, especially serological tumor markers. Methods:Based on the $5 \mathrm{hmC}$-seal technique, the genome-wide-5hydroxymethylcytosine $(5 \mathrm{hmC})$ and gene body $5 \mathrm{hmC}$ content in cfDNA samples from 96 patients were analyzed. Patients were recruited from the Second Affiliated Hospital of Nanchang University; 64 patients had HCC and 32 patients had CLD. Peripheral blood samples were prospectively collected, followed by blood preparation and cfDNA extraction. Independent risk factors associated with HCC were identified by logistic regression, and a diagnostic model was developed and evaluated based on sensitivity, specificity, and correct diagnostic results. Results:The final model revealed sex, age, 5-Hydroxymethylcytosine $(5 \mathrm{hmC})$, and afetoprotein (AFP) as diagnostic indicators in a "discovery" data set. A novel statistical model was formed using the formula: $Z=-18.160+0.081 *$ age $+1.867 * \operatorname{sex}+1.066 *(\log A F P)+1.964 *$ $(\log 5 \mathrm{hmC})$, which had an area under the receiver operating curve of 0.973 . With 0.7636 as the cutoff point, the model had an estimated sensitivity of 0.891 and a specificity of 0.969 . The novel model provides a reliable and stable capacity to predict HCC in patients with CLD. Conclusions: The study findings provide an objective blood serum marker-based model that could enhance the diagnostic efficiency of HCC and form a basis for the early detection of surgically resectable liver cancers in high-risk individuals.

\section{Background}

Hepatocellular carcinoma (HCC) is a serious malignant cancer which ranks as the second leading cause of cancer-related deaths worldwide.[1] HCC is characterized by an insidious onset, strong invasiveness, rapid growth, and high mortality.[2] The poor prognosis of $\mathrm{HCC}$ is due in great extent to the late stage of diagnosis, as symptoms of early stage disease are often vague. In the advanced stages, there are fewer potentially curative treatment options such as liver resection or transplantation. [3] One of the most common causes of HCC is chronic liver disease (CLD). There are many pathogenic factors of chronic liver disease, such as hepatitis B virus (HBV), hepatitis $C$ virus (HCV), and alcoholism, as well as other causes. Greater than 600,000 liver cancers per year result from chronic liver disease, making this a worldwide public health problem.[4] Therefore, common clinical practice guidelines suggest early and periodic examination of CLD patients who have a high risk of developing HCC.[5-8] Furthermore, early diagnosis and initiation of treatment can improve prognosis and are of great significance in reducing mortality from HCC. According to the guidelines of the American Association for the Study of Liver Disease (AASLD), the utilization of alpha-fetoprotein (AFP) and abdominal ultrasound scans are recommended as the primary monitoring tools for early detection of liver cancer.[5] However, AFP and ultrasound have poor specificity 
for the early diagnosis of HCC due to low sensitivity, poor reproducibility, and a high false negative rate, making it an inadequate tool for HCC surveillance.[9-11] Therefore, the identification of novel and more effective diagnostic approaches in HCC is important.

Tumor "liquid biopsy technology" based on cell-free DNA (cfDNA) detection has been a hot spot in cancer research in recent years. Cell-free DNA (cfDNA), which is formed by tumor cells releasing nucleic acid fragments into the bloodstream, is considered a novel tumor molecular marker.[12] CfDNA carries mutations from the original tumor, such as single nucleotide variations, insertion or deletion changes, and epigenetic aberrations, which can be detected in the early stages of tumor development.[13] Therefore, tumor liquid biopsy technology, which is based on cell-free DNA (cfDNA) detection, has emerged as a non-invasive, real-time and comprehensive dynamic method of monitoring tumors while overcoming the limitations of traditional tissue puncture.[14-15]

Epigenetic changes, particularly due to abnormal DNA methylation processes, are closely related to the occurrence and development of tumors. 5-methylcytosine $(5 \mathrm{mC})$ and 5-hydroxymethylcytosine (5hmC) are the fifth and sixth bases on DNA and play a vital role in the epigenetic modification of genes.[16] $5 \mathrm{hmC}$ is an important epigenetic modification which is produced by an enzyme of the TET (ten-eleven translocation) family via oxidation of $5 \mathrm{mC}$. Additionally, the gene distribution of $5 \mathrm{hmc}$ precisely corresponds to the regulation of gene activity, and is more sensitive than $5 \mathrm{mc}$ in reflecting gene expression status.[17] By detecting the distribution of $5 \mathrm{hmC}$ in the genome of cfDNA, gene expression and regulation information can be obtained.[18-19] Therefore, $5 \mathrm{hmC}$ is considered a potentially reliable diagnostic biomarker in human cancers, including HCC. This is due to its tissue and cancer specificity, and more importantly, the rapid development of liquid biopsy technology.[20-22]

In the present study, we utilized a cell-free $5 \mathrm{hmC}$ sequencing method based on selective and sensitive chemical labeling (hMe-seal), to analyze blood samples from a cohort of $64 \mathrm{HCC}$ patients and $32 \mathrm{CLD}$ patients. Our results demonstrated that $5 \mathrm{hmC}$ modifications in the cfDNA of HCC patients exhibited distinct features, with $5 \mathrm{hmC}$ gains significantly enriched compared to those in the cfDNA of CLD controls. In addition, our study showed that the area under the receiver operating characteristic curve (AuROCs) for AFP and $5 \mathrm{hmC}$ were 0.816 (95\% $\mathrm{Cl} 0.731-0.902)$ and 0.891 (95\% $\mathrm{Cl} 0.811-0.972)$ respectively, showing the ability to differentiate between HCC and CLD. A recent study showed that the combination of AFP and $5 \mathrm{hmC}$ may improve the efficiency of tumor detection, especially for the early detection of tumors, by increasing the AuROCs.[23]

Based on this evidence, we determined that an early screening tool for the diagnosis of liver cancer could be developed using two serological measurements, AFP and $5 \mathrm{hmC}$, via establishment of a statistical model. Because diagnostic models require favorable, effective functions for the diagnosis of disease, we carefully collected clinical data to accurately classify the patients according to disease type.

\section{Methods}




\section{Study design and blood samples}

A case-control study was conducted to measure the biomarkers AFP and $5 \mathrm{hmC}$ in blood samples collected between January 2018 and January 2019 at the Second Affiliated Hospital of Nanchang University, Nanchang, China. Samples were taken from patients with HCC or chronic liver disease (CLD) (Table 1). For all patients with liver cancer, the diagnoses were made based on clinical manifestations, pathological examination, imaging examination, and other related examinations according to international guidelines.[24] Additionally, the cancer patients did not undergo surgery, radiofrequency ablation, intervention, radiotherapy, chemotherapy or any other anti-tumor treatments. Patients with incomplete clinical data or other primary tumors were excluded. Blood samples from subjects were collected before any radical treatment according standard operating procedures.[21] The control CLD samples were obtained from patients who were treated for CLD in the infectious diseases department at the same institution. Pathogenic factors of CLD were related to HBV, HCV, alcoholism, or "other" causes. The diagnosis of CLD was based on the analysis of clinical manifestations, liver biopsy and imaging examination. The study protocol was approved by the Medical Ethics committee of the Second Affiliated Hospital of Nanchang University, and all study subjects were provided with written, informed consent. For each subject, the following clinical data were collected: age, gender, race, etiology of liver disease, white blood cell counts (WBC), hemoglobin, total bilirubin (TBil), albumin (ALB), alpha-fetoprotein (AFP), carcinoembryonic antigen (CEA), and 5-Hydroxymethylcytosine (5hmC). The derivation cohort was developed based on these blood samples.

Additional samples from the validation cohort were used to validate the diagnostic model derived from the development cohort. These were obtained between January 2019 and June 2019 at the Tumor Hospital of Jiangxi Province, Nanchang, China. The validation study included 40 subjects; of these, 25 HCC patients and 15 controls with CLD were included in this analysis and the same screening criteria were used to validate the performance of the model (Table 1). For each subject, these clinical data were collected: age, gender, race, etiology of liver disease, white blood cell counts (WBC), hemoglobin, total bilirubin (TBil), albumin (ALB), alpha-fetoprotein (AFP), carcinoembryonic antigen (CEA), and 5Hydroxymethylcytosine $(5 \mathrm{hmC})$. This study was approved by the Medical Ethics committee of the Tumor Hospital of Jiangxi Province. The validation cohort was developed based on these blood samples.

\section{Assays For $5 \mathrm{hmc}$ And Afp}

To access the genome-wide $5 \mathrm{hmC}$ profiles and gains in cfDNA, we used the $5 \mathrm{hmC}$-Seal, a highly sensitive and selective chemical labelling-based sequencing technology developed and optimized by YiBien Technology Co.,Ltd (Shanghai, China). Detailed information about preparation of cfDNA, 5hmC-Seal library construction, sequencing and data processing has been reported in the literature.[21] As AFP is a routine examination for patients with HCC or CLD, measurements were collected via clinical data. No adverse events occurred during this study. 


\section{Statistical analysis}

\section{Model development}

Continuous variables that confirm a normal distribution are represented as mean \pm standard deviation, and categorical variables are represented as frequencies. For univariate and multivariate analyses, logistic regression was employed to calculate the odds ratios (ORs) to evaluate the strength of the correlation with liver cancer. In the multivariable models, age, sex, albumin, bilirubin, 5hmC, AFP, were weighed as risk factors. For statistical analysis, patients with missing data were removed; more than $96 \%$ of cases had complete data on diagnostic model evaluations. Log transformation was performed due to the extreme skew of the data. Logistic regression was based on a complete case study applying a stepwise backward selection method. When the model was established, the Hosmer-Lemeshow test was used to evaluate the goodness of fit for the diagnostic model, and the area under the curve of $5 \mathrm{hmC}$ and AFP was calculated to evaluate the sensitivity, specificity, and optimal threshold of the diagnosis of liver cancer. VIF (variance inflation factor) was used to evaluate whether the research factors had collinearity in the model. Data were analyzed by GraphPad Prism 7.0 (San Diego, CA) and SPSS 22.0 (SPSS, Inc., Chicago, IL). Measurement data are represented as mean \pm standard deviation.

\section{Model Validation}

Model validation was performed in a data set of 40 patients diagnosed with HCC or CLD. Using the model derived from the development cohort, the predictive risk score was obtained to identify patients with and without HCC. This was then compared with the actual diagnosis value. 
Table 1. Characteristics of patients with hepatocellular carcinoma and CLD

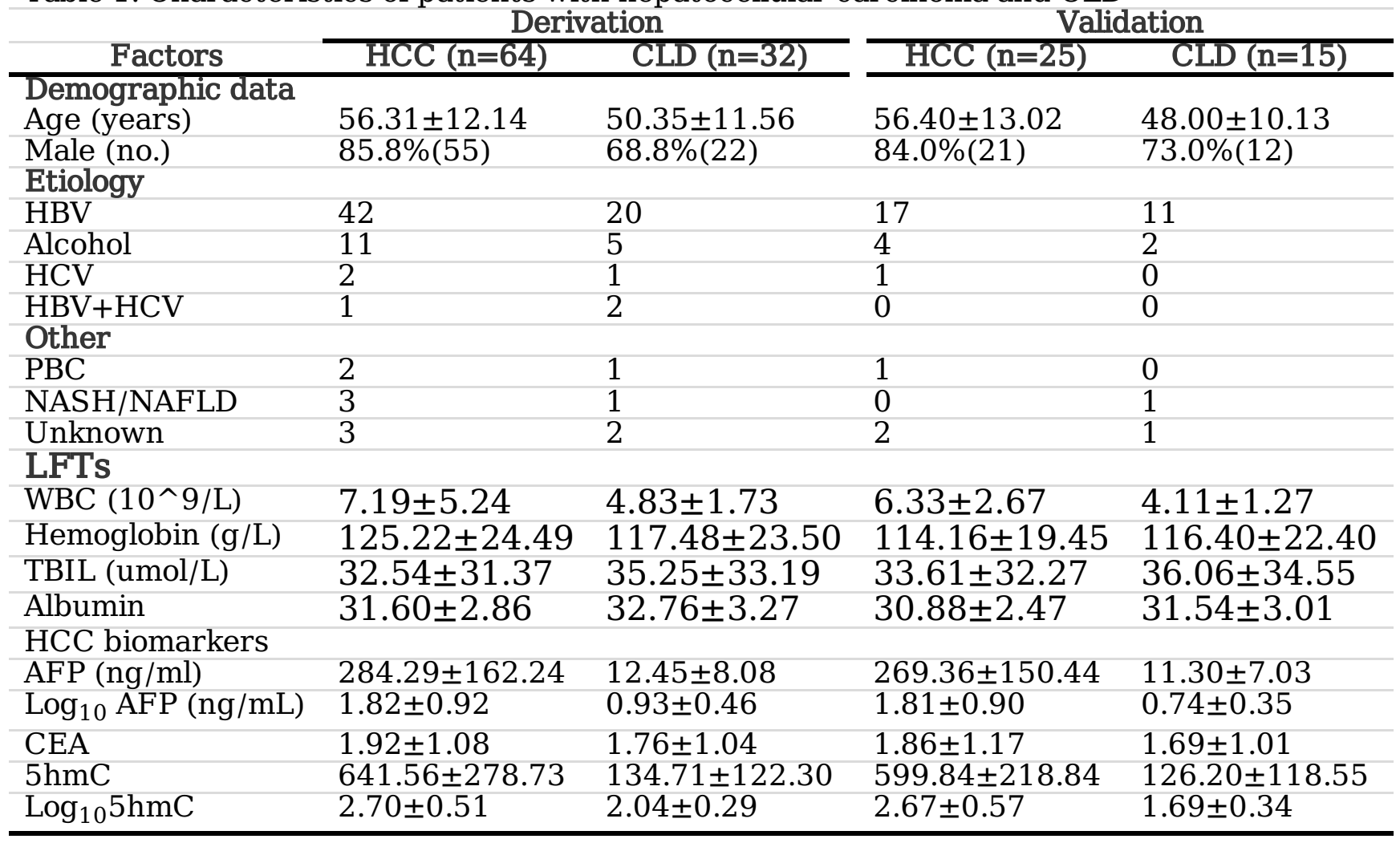

NOTE: For all continuous variables, values are presented either as mean $( \pm S D)$.

Abbreviations: PBC, primary biliary cirrhosis; NASH, nonalcoholic steatohepatitis; NAFLD, nonalcoholic fatty liver disease; WBC, white blood cell counts; TBIL, total bilirubin; AFP, alpha fetoprotein; CEA, carcinoembryonic antigen; 5hmC, 5-Hydroxymethylcytosine.

\section{Results}

\section{Patient characteristics}

Two phases were included in our study (Fig. 1). Characteristics of the patients in two cohorts are summarized in Table 1. The development cohort included blood samples from 64 patients with hepatocellular carcinoma; 61 had tangible evidence of relevant CLD and the presence or absence of underlying CLD could not to confirmed in the remaining 3. The corresponding data for the non-malignant liver disease (CLD) group were $93.8 \%$ and 6.2\%, respectively. Patient mean age in the HCC group was 56.3 years, while the CLD group had a mean age of 50.3. Both groups had a greater proportion of males. Among all the data included in the derivation cohort, data integrity was higher than $98 \%$.

The Validation cohort included 40 patient samples, including 25 patients with HCC and 15 patients with CLD. Among the liver cancer patients, 23 had underlying disease with CLD, and the presence or absence of CLD could not be determined in the other 2. The corresponding data for the non-malignant liver disease (CLD) group were 93.3\%, and 6.7\%, respectively. Patient mean age in the HCC group was 56.4 years, while the CLD group had a mean age of 48.0. Both groups had a greater proportion of males. The 
development and validation cohorts had similar demographic characteristics in terms of mean age and male to female ratio, with most of the patients in the two cohorts exhibiting CLD etiology.

The median values of $\log (\mathrm{AFP}), \log (5 \mathrm{hmC})$ in patients with $\mathrm{HCC}$ was higher than in patients with CLD (Fig. 2A-B). Moreover, the two biomarkers revealed effective discriminatory capacity for distinguishing between liver cancer and CLD patients (auROCs: $\log ($ AFP) 0.816, $\log (5 \mathrm{hmC})$ 0.891; Fig. 3;

Supplementary Table 3).

\section{Model Performance In The Development Cohort}

Based on the discovery data set, 4 variables were included in the statistical diagnostic model: $\log ($ AFP), $\log (5 \mathrm{hmC})$, and age and sex, which were the most important factors for identifying patients with HCC (Supplementary Table 2). The diagnostic model for identifying patients with HCC used the following formula: $Z=-18.160+0.081 *$ age $+1.867 * \operatorname{sex}+1.066 *(\log A F P)+1.964 *(\log 5 \mathrm{hmC})$

\section{Correlation Between Risk Factors}

Because four variables were contained in the diagnostic model, the occurrence of multiple collinearity was possible, which can affect the fit of the diagnostic model. Therefore, the variance inflation factor was used to test for multicollinearity in the regression model. The obtained coefficient of variance inflation was 1.164 for age, 1.034 for sex, 1.187 for AFP, and 1.351 for $5 \mathrm{hmC}$, indicating that there was no multicollinearity between variables. Therefore, it was considered reasonable to keep these risk factors in the model

\section{Goodness-of-fit Test And The Auroc}

The Hosmer-Lemeshow goodness-of-fit statistical analysis was used to assess the goodness-of-fit of the diagnostic model. The results showed that $P=0.778, \chi^{2}=4.805$, indicating that there was no significant difference between the predicted diagnosis and actual diagnosis. A receiver operating characteristic curve analysis was performed on the diagnostic model, and the results showed that the area under the receiver operating characteristic (auROCs) of the model for predicting the diagnosis of patients with HCC in the CLD individuals was $0.973,95 \%$ (0.945-1.000), (Fig. 3; Supplementary Table 3), indicating that the new model had sufficient discrimination ability.

\section{Model Performance Of The External Validation Cohort}

\section{Validation cohort}


In the validation cohort, data from 40 patients were collected to assess the discriminating power of the diagnostic model using the above formula. $P$ values were calculated for each individual patient. Using 0.7636 as the cutoff point, when the $p$ value was $>0.7636$, patients were considered to have HCC and when the $p$ value was $<0.7636$, they were considered to have a normal CLD. The $p$ values of 21 patients were greater than 0.7636 and 19 patients had $p$ values below 0.7636 . Among the 21 patients who were considered to have HCC according to the diagnostic model, 19 were confirmed to have HCC. Among the 19 patients who were considered to have no HCC according to the diagnostic model, 13 were confirmed to have a normal CLD. Therefore, the sensitivity and specificity of the model in the validation cohort were $76.00 \%(19 / 25)$ and $86.67 \%$ (13/15), respectively (Supplementary Table 4).

\section{Discussions}

HCC is a severe malignancy with high mortality worldwide.[25] Although advanced surgical techniques and radiation therapy have recently been proposed, the survival of patients with HCC remains poor.[2627] The above clinical characteristics highlight the need for more efforts in the prevention and early detection of HCC.[5-7] In the current study, we developed a model based on simple serological biomarkers which had high-efficiency for diagnosing the presence or absence of HCC in CLD patients. This method provides a novel and simple way to diagnose HCC, allowing for early intervention in these high-risk patients.

Measurement of serum AFP and abdominal ultrasound examination are widely used as screening tools for the early detection of HCC in chronic liver disease patients.[28] However, the diagnostic efficiency of these methods remains poor.[5] Studies have confirmed that some patients with liver injury and embryogenic tumors also have elevated serum AFP levels, while ultrasound detection has different degrees of missed diagnosis and misdiagnosis.[29] Therefore, finding better tumor markers is necessary for the diagnosis of early liver cancer.

Recent studies have shown that $5 \mathrm{hmC}$, an intermediate product in the process of cytosine demethylation, exerts a significant influence in the regulation of gene expression and is considered to be an effective epigenetic biomarker for tumor diagnosis or prognosis.[21] For example, Zhang et al., detected the genome wide distribution of 5hmC in cfDNA from 66 patients with NSCLC and 67 healthy individuals, identifying the $5 \mathrm{hmC}$ signatures of cfDNA with high sensitivity and specificity for NSCLC.[30] In a prospective study of 1204 patients with HCC and 392 high-risk Chinese patients with chronic hepatitis or liver cirrhosis, Cai et al., demonstrated the $5 \mathrm{hmC}$-Seal approach to be clinically useful, outperforming AFP, with the potential to be a novel class of epigenetic biomarkers.[23] In addition, the development of highly sensitive $5 \mathrm{hmC}$ markers in cfDNA could serve as a promising noninvasive approach for HCC diagnosis for the general population.[21] In this study, based on a sensitive $5 \mathrm{hmC}$-Seal method, the profiles of cellfree $5 \mathrm{hmC}$ in the blood of HCC patients and CLD subjects were obtained. This method exhibited higher sensitivity and specificity compared with AFP. Even at low or negative AFP values, there remained significant discriminating capacity. 
Ideal tumor marker detection requires high sensitivity, early detection of tumor patients, high specificity, and organ specificity.[31] The detection of single tumor markers could not satisfy these factors, therefore, a combination of multiple serum tumor and risk factors associated with cancer would be more clinically useful for the early diagnosis of tumors.[31-32] Using logistic regression, we identified the independent factors associated with hepatocellular carcinoma and elucidated the relationship between HCC and these factors. The results were a good combination of these independent clinical indicators and provide a model established and evaluated based on sensitivity, specificity, and correct diagnostic proportions.

The current study findings have not fully confirmed or elucidated the specific mechanisms of liver cancer. However, epidemiological studies have confirmed that CLD is an important risk factor for liver cancer, and more than $70 \%$ of liver cancer is caused by CLD.[33] CLD patients are a high-risk group for the occurrence of liver cancer, and this has been reported to be more common among elderly patients. [4] This finding is consistent with the results of our study, which indicated that age was an important factor for identifying patients with HCC. Further, there is a significant gender difference in HCC, and the incidence of male HCC is more than twice that of female HCC. This study finding was also consistent with many reports in the literature.[34-36] According to the logistic regression analysis, AFP and 5hmC also indicated the presence of HCC. Therefore, the model is based on four objective variables: age, sex, AFP, 5hmC. In the model development, the variance inflation factor test confirmed that there was no multicollinearity in the regression model, and the goodness-of-fit testing confirmed the rationality of the diagnostic model based on the four objective variables.

The optimal way to validate a diagnostic model is to assess its performance in an independent patient cohort. We used a cohort of patients diagnosed with HCC or CLD who were treated at a single hospital from January 2019 to June 2019. The model performed well in the validation cohort compared with the derivation cohort (sensitivity: $76.00 \%$, specificity: $86.67 \%$ vs sensitivity: $89.10 \%$, specificity: $96.90 \%$ ). The model had comparable discrimination ability in the validation cohort, demonstrating that this model had an acceptable and stable discrimination ability for diagnosing liver cancer in patients with CLD, and indicating that this model is valuable for identifying HCC.

Early diagnosis of and intervention in high-risk populations is regarded an effective treatment for HCC. [37-38] Various techniques have been used clinically to detect tumor information, including methods based on liquid biopsy such as early liquid biopsy assays targeting exosomes or noncoding RNAs.[3940] Though promising, these approaches do not offer satisfactory performance in terms of specificity and sensitivity suitable for a clinical scale. Based on a cell-free $5 \mathrm{hmC}$ sequencing method, we detected an increased $5 \mathrm{hmC}$ gain in blood samples from HCC patients compared with CLD controls. Combined with AFP levels, sex, and age, we developed a diagnostic model for distinguishing HCC in high risk individuals with CLD, showing improved performance compared with normal diagnostic markers. Additionally, given its non-invasiveness and the tissue-specificity of $5 \mathrm{hmC}$, the model may also serve as a convenient tool for detection in the general population. 
Because this study had a limited number of cases and was based on a Chinese patient population with a high CLD background, further validation in prospective studies will be necessary to demonstrate the generalizability of the results to other populations and geographical regions. With further research, we believe this model will have superior performance and universality for HCC diagnosis.

\section{Conclusions}

In summary, we have identified important factors for HCC in CLD patients, and developed and validated a novel, non-invasive diagnostic model for surgically resectable HCC using the highly sensitive $5 \mathrm{hmC}$-Seal assay. Compared with the pathological examination, preoperative blood testing is convenient, widely available, and routinely performed in clinical practice. This model may provide a useful tool for the preoperative diagnosis of and early intervention in HCC, which could lay the foundation for developing a future pan-cancer, non-invasive screening tool.

\section{Abbreviations}

HCC: Hepatocellular carcinoma; CLD: Chronic liver disease; 5hmC: 5-Hydroxymethylcytosine; HBV: Hepatitis B virus; HCV: Hepatitis C virus; AASLD: American Association for the Study of Liver Disease; FAFP: Alpha-fetoprotein; cfDNA: Cell-free DNA; 5mC: 5-methylcytosine; TET: Ten-eleven translocation; hMe-seal: Hydroxymethyl selective chemical labelling; AuROCs: Area under the receiver operating characteristic curve; WBC: White blood cell counts; Tbil: Total bilirubin; ALB: Albumin; AFP: Alphafetoprotein; CEA: Carcinoembryonic antigen; ORs: Odds ratios.

\section{Declarations}

\section{Ethics approval and consent to participate}

The study protocol was approved by the Medical Ethics committee of the Second Affiliated Hospital of Nanchang University, and all study subjects were provided with written, informed consent.

\section{Consent for publication}

Not applicable.

\section{Availability of data and materials}

The datasets obtained in the study are available from the corresponding author upon reasonable request.

\section{Competing interests}

The authors declare that they have no conflict of interests.

\section{Funding}


This investigation was funded the Key Foundation of Jiangxi Provincial Science and Technology Department (No. 20171ACB21064), the Youth Science Foundation of Jiangxi Provincial Science and Technology Department (No. 20151BAB205105). The funders had no role in the design of the study and collection, analysis, and interpretation of data and in producing the manuscript.

\section{Author contributions}

JG, C-YX, XY, Y-BX, X-GP, X-BY, D-JL and FZ designed the study. JG and C-YX collated the data, conducted data analyses, and produced the initial draft of the manuscript. XY, Y-BX, X-GP, X-BY, D-JL and FZ contributed to drafting the manuscript. All authors have read and approved the final submitted manuscript.

\section{Acknowledgements}

We thank YiBien Technology Company for the technical assistance with $5 \mathrm{hmC}$-seal sequencing and the members of our research team for their contributions. We thank LetPub (www.letpub.com) for its linguistic assistance during the preparation of this manuscript.

\section{References}

1. Vilgrain V, Pereira H, Assenat E, Guiu B, llonca AD, Pageaux GP, Sibert A, Bouattour M, Lebtahi R, Allaham W: Efficacy and safety of selective internal radiotherapy with yttrium-90 resin microspheres compared with sorafenib in locally advanced and inoperable hepatocellular carcinoma (SARAH): an open-label randomised controlled phase 3 trial. Lancet Oncol 2017, 18:1624-1636.

2. Takeaki I, Kiyoshi H, Taku A, Michiro T, Yosuke I, Keiji S, Hiroshi I, Yasuhiko S, Norihiro K, Masatoshi M: Neither multiple tumors nor portal hypertension are surgical contraindications for hepatocellular carcinoma. Gastroenterology 2008, 134(7):1908-1916.

3. Hasegawa K, Aoki T, Ishizawa T, Kaneko J, Sakamoto Y, Sugawara Y, Kokudo N: Comparison of the Therapeutic Outcomes Between Surgical Resection and Percutaneous Ablation for Small Hepatocellular Carcinoma. Ann Surg Oncol 2014, 21(3 Supplement):348-355.

4. Yang JD, Hainaut P, Gores GJ, Amadou A, Plymoth A, Roberts LR: A global view of hepatocellular carcinoma: trends, risk, prevention and management. Nature reviews Gastroenterology \& hepatology 2019.

5. Heimbach JK, Kulik LM, Finn RS, Sirlin CB, Abecassis MM, Roberts LR, Zhu AX, Murad MH, Marrero JA: AASLD guidelines for the treatment of hepatocellular carcinoma. Hepatology (Baltimore, Md) 2018, 67(1):358-380.

6. Tsochatzis E, Meyer T, O'Beirne J, Burroughs AK: Transarterial chemoembolisation is not superior to embolisation alone: the recent European Association for the Study of the Liver (EASL) - European Organisation for Research and Treatment of Cancer (EORTC) guidelines. European journal of cancer (Oxford, England : 1990) 2013, 49(6):1509-1510. 
7. Kokudo N, Takemura N, Hasegawa K, Takayama T, Kubo S, Shimada M, Nagano H, Hatano E, Izumi N, Kaneko S et al: Clinical Practice Guidelines for Hepatocellular Carcinoma: The Japan Society of Hepatology 2017 (4th JSH-HCC Guidelines) a 2019 update. Hepatology research : the official journal of the Japan Society of Hepatology 2019.

8. Swarm RA, Paice JA, Anghelescu DL, Are M, Bruce JY, Buga S, Chwistek M, Cleeland C, Craig D, Gafford E et al: Adult Cancer Pain, Version 3.2019, NCCN Clinical Practice Guidelines in Oncology. Journal of the National Comprehensive Cancer Network : JNCCN 2019, 17(8):977-1007.

9. Atiq O, Tiro J, Yopp AC, Muffler A, Marrero JA, Parikh ND, Murphy C, McCallister K, Singal AG: An assessment of benefits and harms of hepatocellular carcinoma surveillance in patients with cirrhosis. Hepatology (Baltimore, Md) 2017, 65(4):1196-1205.

10. El-Bahrawy M: Alpha-fetoprotein-producing non-germ cell tumours of the female genital tract. European journal of cancer (Oxford, England : 1990) 2010, 46(8):1317-1322.

11. Sterling RK, Wright EC, Morgan TR, Seeff LB, Hoefs JC, Di Bisceglie AM, Dienstag JL, Lok AS: Frequency of elevated hepatocellular carcinoma (HCC) biomarkers in patients with advanced hepatitis C. The American journal of gastroenterology 2012, 107(1):64-74.

12. Sun K, Jiang P, Chan KC, Wong J, Cheng YK, Liang RH, Chan WK, Ma ES, Chan SL, Cheng SH et al: Plasma DNA tissue mapping by genome-wide methylation sequencing for noninvasive prenatal, cancer, and transplantation assessments. Proceedings of the National Academy of Sciences of the United States of America 2015, 112(40):E5503-5512.

13. Schwarzenbach $\mathrm{H}$, Hoon DS, Pantel $\mathrm{K}$ : Cell-free nucleic acids as biomarkers in cancer patients. Nature reviews Cancer 2011, 11(6):426-437.

14. Pezzuto F, Buonaguro L, Buonaguro FM, Tornesello ML: The Role of Circulating Free DNA and MicroRNA in Non-Invasive Diagnosis of HBV- and HCV-Related Hepatocellular Carcinoma. Int J Mol Sci 2018, 19(4).

15. Lu CY, Chen SY, Peng HL, Kan PY, Chang WC, Yen CJ: Cell-free methylation markers with diagnostic and prognostic potential in hepatocellular carcinoma. Oncotarget 2017, 8(4):6406-6418.

16. Branco MR, Ficz G, Reik W: Uncovering the role of 5-hydroxymethylcytosine in the epigenome. Nat Rev Genet 2011, 13(1):7-13.

17. Tahiliani M, Koh KP, Shen Y, Pastor WA, Bandukwala H, Brudno Y, Agarwal S, lyer LM, Liu DR, Aravind $\mathrm{L}$ et al: Conversion of 5-methylcytosine to 5-hydroxymethylcytosine in mammalian DNA by MLL partner TET1. Science (New York, NY) 2009, 324(5929):930-935.

18. Mariani CJ, Madzo J, Moen EL, Yesilkanal A, Godley LA: Alterations of 5-hydroxymethylcytosine in human cancers. Cancers 2013, 5(3):786-814.

19. Song CX, Yin S, Ma L, Wheeler A, Chen Y, Zhang Y, Liu B, Xiong J, Zhang W, Hu J et al: 5Hydroxymethylcytosine signatures in cell-free DNA provide information about tumor types and stages. Cell research 2017, 27(10):1231-1242.

20. Zeng C, Stroup EK, Zhang Z, Chiu BC, Zhang W: Towards precision medicine: advances in 5hydroxymethylcytosine cancer biomarker discovery in liquid biopsy. Cancer communications 
(London, England) 2019, 39(1):12.

21. Li W, Zhang X, Lu X, You L, Song Y, Luo Z, Zhang J, Nie J, Zheng W, Xu D et al: 5Hydroxymethylcytosine signatures in circulating cell-free DNA as diagnostic biomarkers for human cancers. Cell research 2017, 27(10):1243-1257.

22. Gao P, Lin S, Cai M, Zhu Y, Song Y, Sui Y, Lin J, Liu J, Lu X, Zhong Y et al: 5-Hydroxymethylcytosine profiling from genomic and cell-free DNA for colorectal cancers patients. Journal of cellular and molecular medicine 2019, 23(5):3530-3537.

23. Cai J, Chen L, Zhang Z, Zhang X, Lu X, Liu W, Shi G, Ge Y, Gao P, Yang Y et al: Genome-wide mapping of 5-hydroxymethylcytosines in circulating cell-free DNA as a non-invasive approach for early detection of hepatocellular carcinoma. Gut 2019.

24. Bruix J, Sherman M: Management of hepatocellular carcinoma: An update. Hepatology (Baltimore, Md) 2011, 53(3):1020-1022.

25. Bray F, Ferlay J, Soerjomataram I, Siegel RL, Torre LA, Jemal A: Global cancer statistics 2018: GLOBOCAN estimates of incidence and mortality worldwide for 36 cancers in 185 countries. CA: a cancer journal for clinicians 2018, 68(6):394-424.

26. Ishizawa T, Hasegawa K, Aoki T, Takahashi M, Inoue Y, Sano K, Imamura H, Sugawara Y, Kokudo N, Makuuchi M: Neither multiple tumors nor portal hypertension are surgical contraindications for hepatocellular carcinoma. Gastroenterology 2008, 134(7):1908-1916.

27. Ho MC, Hasegawa K, Chen XP, Nagano H, Lee YJ, Chau GY, Zhou J, Wang CC, Choi YR, Poon RT et al: Surgery for Intermediate and Advanced Hepatocellular Carcinoma: A Consensus Report from the 5th Asia-Pacific Primary Liver Cancer Expert Meeting (APPLE 2014). Liver cancer 2016, 5(4):245-256.

28. Gupta S, Bent S, Kohlwes J: Test characteristics of alpha-fetoprotein for detecting hepatocellular carcinoma in patients with hepatitis C. A systematic review and critical analysis. Annals of internal medicine 2003, 139(1):46-50.

29. Sell S: AFP as a marker for liver cell injury: differentiation of tumor growth, hepatotoxicity, and carcinogenesis. UCLA forum in medical sciences 1978, 20:51-58.

30. Zhang J, Han X, Gao C, Xing Y, Qi Z, Liu R, Wang Y, Zhang X, Yang YG, Li X et al: 5-Hydroxymethylome in Circulating Cell-free DNA as A Potential Biomarker for Non-small-cell Lung Cancer. Genomics, proteomics \& bioinformatics 2018, 16(3):187-199.

31. Kobayashi T: A blood tumor marker combination assay produces high sensitivity and specificity for cancer according to the natural history. Cancer medicine 2018, 7(3):549-556.

32. Choi JY, Jung SW, Kim HY, Kim M, Kim Y, Kim DG, Oh EJ: Diagnostic value of AFP-L3 and PIVKA-II in hepatocellular carcinoma according to total-AFP. World journal of gastroenterology 2013, 19(3):339346.

33. Tinkle CL, Haas-Kogan D: Hepatocellular carcinoma: natural history, current management, and emerging tools. Biologics : targets \& therapy 2012, 6:207-219.

34. Hemken PM, Sokoll LJ, Yang X, Dai J, Elliott D, Gawel SH, Lucht M, Feng Z, Marrero JA, Srivastava S et al: Validation of a novel model for the early detection of hepatocellular carcinoma. Clinical 
proteomics 2019, 16:2.

35. Zhang S, He LL, Wang XH, Dang ZB, Liu XL, Li MG, Wang XB, Yang ZY: A novel scoring model for predicting mortality risk in patients with cirrhosis and hepatorenal syndrome. European journal of gastroenterology \& hepatology 2018, 30(8):938-943.

36. Johnson PJ, Pirrie SJ, Cox TF, Berhane S, Teng M, Palmer D, Morse J, Hull D, Patman G, Kagebayashi $\mathrm{C}$ et al: The detection of hepatocellular carcinoma using a prospectively developed and validated model based on serological biomarkers. Cancer epidemiology, biomarkers \& prevention : a publication of the American Association for Cancer Research, cosponsored by the American Society of Preventive Oncology 2014, 23(1):144-153.

37. Kanwal F, Singal AG: Surveillance for Hepatocellular Carcinoma: Current Best Practice and Future Direction. Gastroenterology 2019, 157(1):54-64.

38. Singal A, Volk ML, Waljee A, Salgia R, Higgins P, Rogers MA, Marrero JA: Meta-analysis: surveillance with ultrasound for early-stage hepatocellular carcinoma in patients with cirrhosis. Alimentary pharmacology \& therapeutics 2009, 30(1):37-47.

39. Pardini B, Sabo AA, Birolo G, Calin GA: Noncoding RNAs in Extracellular Fluids as Cancer Biomarkers: The New Frontier of Liquid Biopsies. Cancers 2019, 11(8).

40. He M, Zeng Y: Microfluidic Exosome Analysis toward Liquid Biopsy for Cancer. Journal of laboratory automation 2016, 21(4):599-608.

\section{Supplementary Table Legends}

supplementary Table2 Parameter estimates (SE) and OR (95\% Cl) of variables based on the model. supplementary Table3 Diagnostic performance of biomarkers alone and the model performance.

supplementary Table4 Sensitivity and specificity analysis in the validation cohort.

\section{Figures}




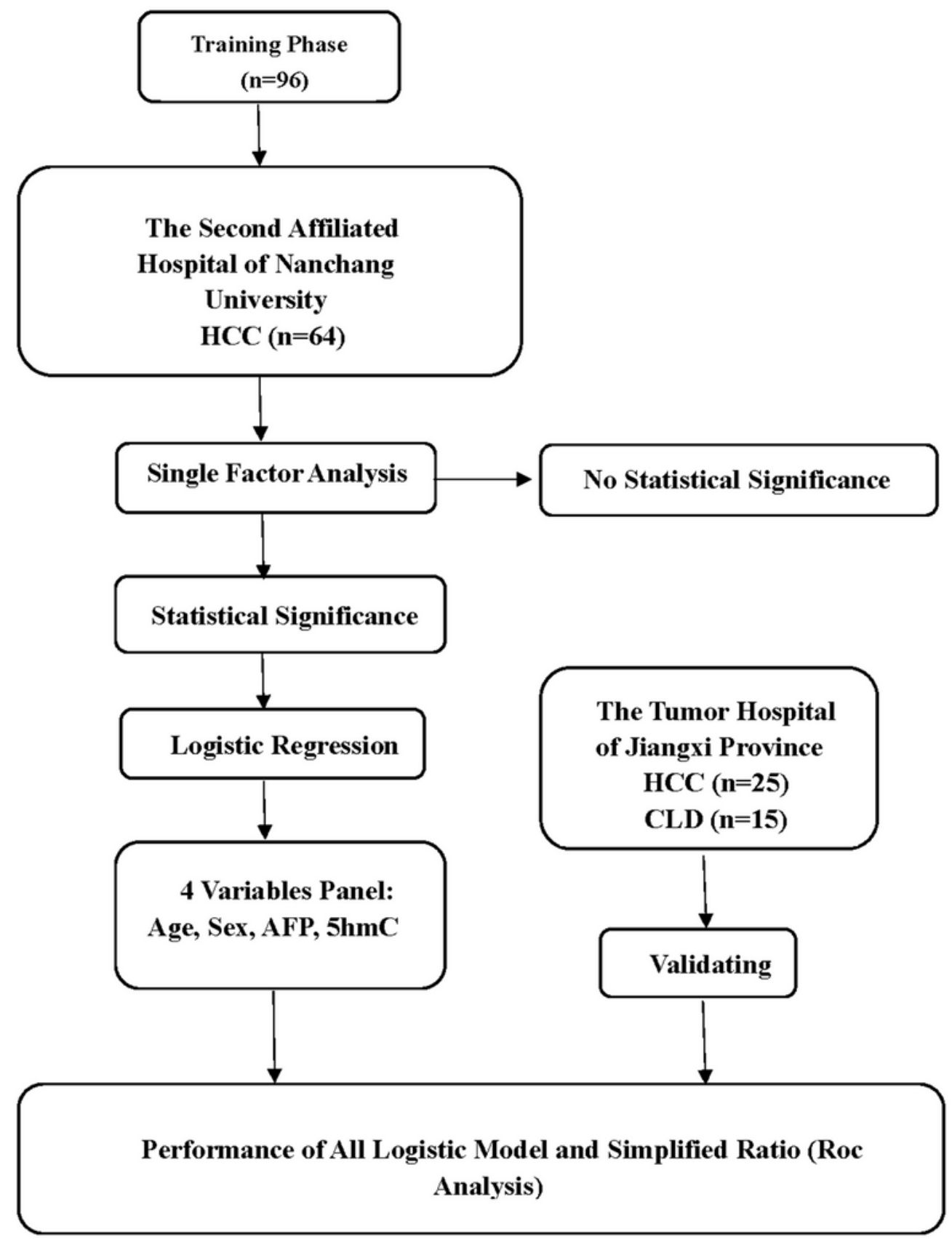

Figure 1. Study design. HCC, Hepatocellular carcinoma; CLD, chronic liver disease; AFP, alpha-fetoprotein; 5hmC, 5-hydroxylmethylcytosine; ROC, receiver operating characteristics.

Figure 1

Study design. HCC, Hepatocellular carcinoma; CLD, chronic liver disease; AFP, alpha-fetoprotein; 5hmC, 5hydroxylmethylcytosine; ROC, receiver operating characteristics. 
A

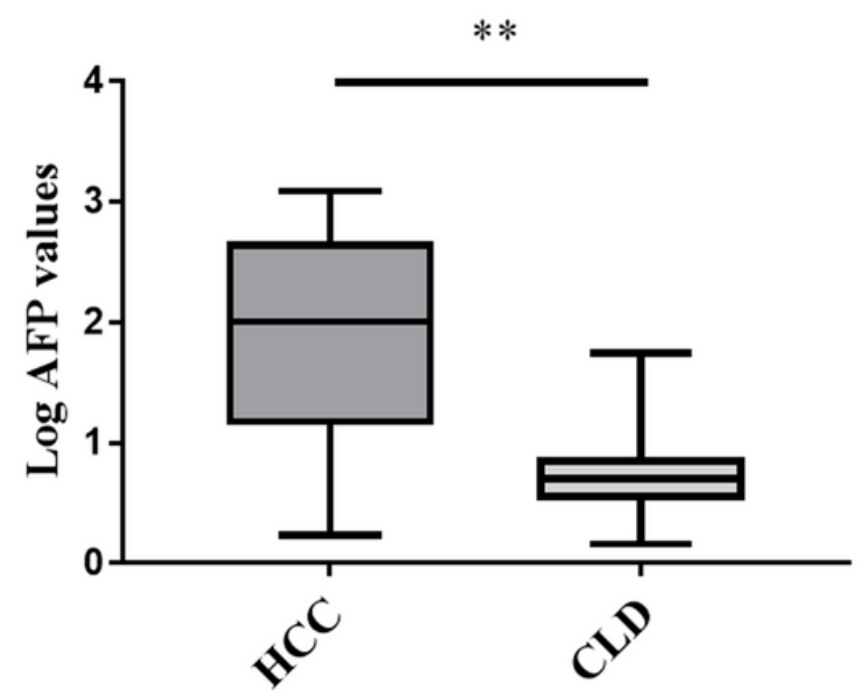

B

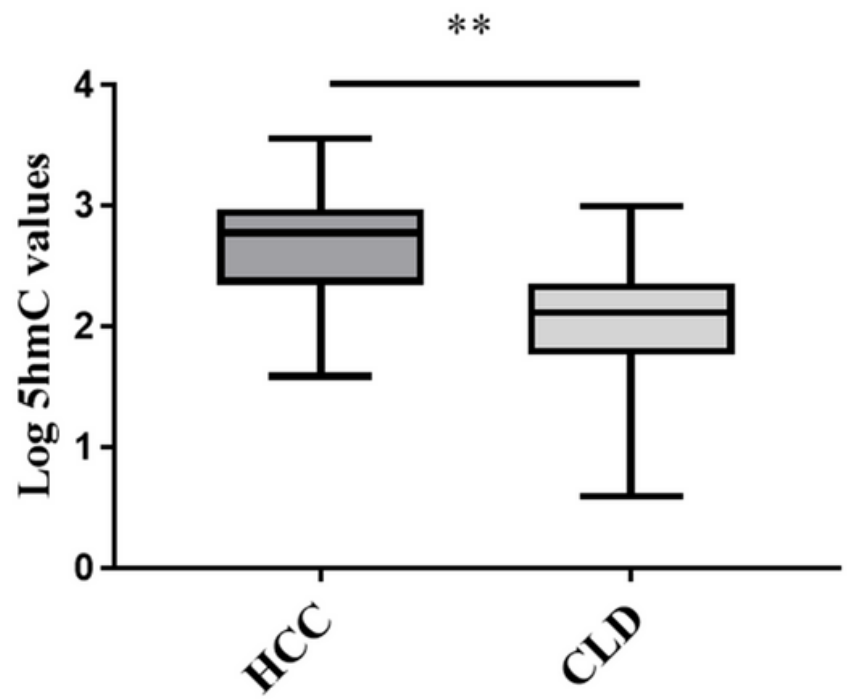

Figure 2

(A) Log AFP values in the HCC and CLD patients. (B) Log $5 \mathrm{hmC}$ values in the HCC and CLD patients. **P $<0.01$

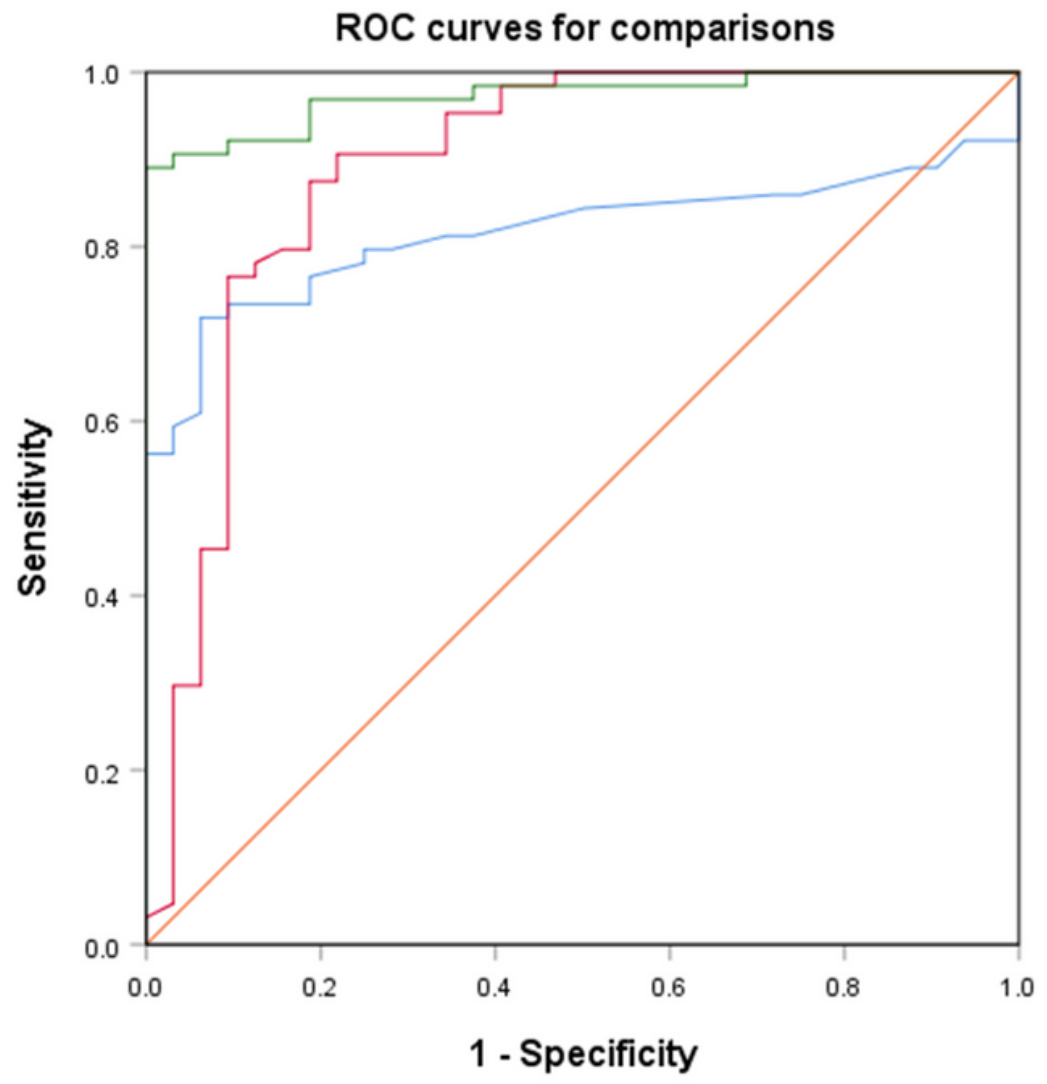

Source of the curve

$-\log (\mathrm{AFP})$
$-\log (5 \mathrm{hmC})$

- Model

Reference line

Figure 3 
The ROC curves for Log AFP, log $5 \mathrm{hmC}$ and model for predicting the diagnosis of patients with HCC in the CLD individuals.

\section{Supplementary Files}

This is a list of supplementary files associated with this preprint. Click to download.

- SupplementaryTable3.docx

- SupplementaryTable4.docx

- SupplementaryTable2.docx 